\title{
TOPOLOGICAL SPACES WHICH ADMIT UNISOLVENT SYSTEMS
}

BY

JOHN A. LUTTS, S. J.

Introduction. We begin with a definition. Let $X$ be a topological space and let $\mathscr{C}(X)$ be the set of all continuous functions which map $X$ into $R$, the real numbers. Let $\mathscr{F}=\left\{f_{1}, \cdots, f_{n}\right\}$ be a set of $n$ distinct functions in $\mathscr{C}(X)$ such that each nonzero linear combination of these functions over $R$ has at most $n-1$ zeros in $X$. It is clear that this property of $\mathscr{F}$ is equivalent to the property that given any set of $n$ distinct points $\left\{x_{1}, \cdots, x_{n}\right\}$ in $X$, the matrix $\left\|f_{i}\left(x_{j}\right)\right\|$ has an inverse. We may call such a set $\mathscr{F}$ a real-valued unisolvent system of order $n$ on $X$, or for brevity, an $n-R-U$ system on $X\left({ }^{1}\right)$. For examples, confer (1.6) and (2.5) below.

The purpose of this paper is to characterize topologically those spaces which admit $n-R-U$ systems. It should be noted that any nonempty topological space of less than $n$ points has an $n-R-U$ system and that any nonempty topological space whatsover has a $1-R-U$ system. Consequently in this paper we shall consider only spaces with at least $n$ points, where $n \geqq 2$.

A summary of this problem's background is useful. For this the following definition is needed. Let $\mathscr{M}$ be an $n$-dimensional vector $R$-subspace of $\mathscr{C}_{0}(X)$, where $X$ is a locally compact Hausdorff space and $\mathscr{C}_{0}(X)$ is the vector $R$-subspace of $\mathscr{C}(X)$ containing all functions which "vanish at infinity" (i.e., all functions $f$ in $\mathscr{C}(X)$ such that for each $\varepsilon>0$, the set $\{x \in X:|f(x)| \geqq \varepsilon\}$ is compact). $\mathscr{M}$ is said to be a Haar subspace of $\mathscr{C}_{0}(X)$ if given any $f$ in $\mathscr{C}_{0}(X)$ there is a unique $g_{f}$ in $\mathscr{M}$ such that

$$
\sup _{x \in X}\left|f(x)-g_{f}(x)\right| \leqq \sup _{x \in X}|f(x)-g(x)|
$$

for all $g$ in $\mathscr{M}$. Phelps [12] proved that a set of $n$ linearly independent functions (over $R$ ) in $\mathscr{C}_{0}(X)$ span such a subspace $\mathscr{M}$ if and only if the set is, in our terminology, an $n-R-U$ system on $X$. Haar [7] had proved this equivalence when $X$ is a compact subset of some Euclidean space. Using Haar's result, Mairhuber [10] proved that for such a compact space (with at least $n$ points, $n \geqq 2$ ): (a) If $X$ admits an $n$-dimensional Haar subspace in $\mathscr{C}_{0}(X)$, then $X$ is homeomorphic to a

Received by the editors February 16, 1963.

(1) For the name unisolvent, confer [11, p. 1] (where its meaning is somewhat different) and especially [3]. Other terminology used in this area: in [13] and [14], [X; $\mathscr{F}]$ is called a real Haar system; in [6], if $\mathscr{F}$ is linearly independent over $R$, the span of $\mathscr{F}$ over $R$ in $\mathscr{C}(X)$ is called a linear n-parameter family; in [2] and [10], $\mathscr{F}$ is called a Chebychev system. 
closed subset of a one-sphere. If in addition $n$ is even, then this subset must be proper. (b) Conversely, if $X$ is topologically imbeddable into a one-sphere, then $X$ admits an $n$-dimensional Haar subspace in $\mathscr{C}_{0}(X)$ (provided that $n$ is odd when $X$ is a one-sphere $\left({ }^{2}\right)$. In view of his extension of Haar's result, Phelps asked whether a similar characterization could be found for locally compact Hausdorff spaces.

This paper resulted from an attempt to answer that question. But, taking our cue from the proofs that have been given of Mairhuber's result (cf. footnote 2), we made the $n-R-U$ system and not the Haar subspace the focal point of organization (since all the topological information is derived from it). Moreover, we obtain the answer by way of corollary to results more general and of more immediate interest. Specifically, we prove that for a topological space with at least $n$ points, $n \geqq 2$,

(a) If there is an $n-R-U$ system on $X$, then there is a continuous one-one map of $X$ into a one-sphere. Conversely, if there is a continuous one-one map of $X$ into a one-sphere, then there is an $n-R-U$ system on $X$ (provided that $n$ is odd when all such maps are onto).

(b) The following conditions are each sufficient for $X$ to be topologically imbeddable into a one-sphere when it has an $n-R-U$ system:

(1) $X$ is connected and is either locally connected or locally compact.

(2) $X$ is locally compact and $\sigma$-compact.

(c) Since $\sigma$-compactness obtains in a space any member of whose $n-R-U$ system "vanishes at infinity," Phelps' question is answered in the affirmative and the characterization is identical with that given above by Mairhuber for compact spaces.

The author wishes to take this opportunity to express his gratitude to Professor Chung-Tao Yang for his help and encouragement in this investigation.

1. General characterization. In this section we give a necessary and sufficient condition for a topological space to have an $n-R-U$ system. The proof of our result depends on a generalization of certain key concepts and propositions found in the various proofs of Mairhuber's result (cf. footnote 2). We list them here noting that in all of them the space $X$ is assumed to be compact Hausdorff and to have at least $n$ points and an $n-R-U$ system ( $n \geqq 2)$. First, for each open set $U$ in $X$ containing at least $n-2$ points, there is a homeomorphism $f_{U}$ of $X-U$ into a one-sphere. Secondly, for each connected subset $Y$ of $f_{U}(X-U), f_{U}^{-1}(Y)$ is connected. Thus each component of $X$, when not a point, is a finite union of arcs and contains no triods. Lastly, if $X$ contains a one-sphere, $X$ is that onesphere.

(2) Proofs of Haar's theorem under the assumption that $X$ is a compact Hausdorff space are given in [1] and [12], while Mairhuber's result in this broader context is proved in [6], [14] and [15]. Similar approximation theory results and corresponding topological results for complexvalued functions are given in [14]. 
A few preparatory lemmas are needed. For the sake of convenience we introduce the following definition.

(1.1) Definition. Let $X$ be a topological space. Then $X=A_{1} \cup A_{2}$ is called a nontrivial separation if $A_{i} \neq \varnothing$, for $i=1,2$, and $\left(\overline{A_{1}} \cap A_{2}\right) \cup\left(A_{1} \cap \overline{A_{2}}\right)=\varnothing$.

(1.2) LEMMA. Let $X$ be a connected topological space and let $f$ be a continuous one-one map of $X$ into $R$. Then for each connected subset $Y$ of $f(X), f^{-1}(Y)$ is connected.

Proof. We may assume that $X$ contains more than a single point. We first consider the special case where $Y$ is a component of $f(X)-\{f(b)\}$ for some point $b$ in $X$.

We first assume that $f(b)$ is an end point of $f(X)$. Our claim in this case is that $X-\{b\}$ is connected. Assuming that it is not, we let $X-\{b\}=A_{1} \cup A_{2}$ be a nontrivial separation. Then, since for $i=1,2, A_{i} \cup\{b\}$ is connected and $f(b)$ is the only point common to their images in $R$, it is impossible that $f(b)$ be the end point of $f(X)$. This contradiction proves the claim.

Next we assume that $f(b)$ is not an end point of $f(X)$ and let $f(X)-\{f(b)\}$ $=A_{1} \cup A_{2}$ be the nontrivial separation. Our claim in this case is that $f^{-1}\left(A_{i}\right)$ is connected, $i=1,2$. Now since $X-\{b\}=f^{-1}\left(A_{1}\right) \cup f^{-1}\left(A_{2}\right)$ is a nontrivial separation, $f^{-1}\left(A_{i}\right) \cup\{b\}$ is connected. Then, since $f(b)$ is the end point of $A_{i} \cup\{f(b)\}$, our claim is a corollary to the previous case.

Since $f(X)$ is an interval in $R$, one may readily obtain the general result by considering the various types of subintervals of $f(X)$ and by using the results of the two previous paragraphs.

Q.E.D.

(1.3) COROLlary. Let $X$ be a topological space such that any continuous one-one map of $X$ into a one-sphere $S^{1}$ is onto. Let $f$ be one such map. Then for every connected subset $Y$ of $S^{1}, f^{-1}(Y)$ is connected.

Proof. From the hypothesis on $X$ one can easily see that $X$ itself is connected. We shall then first consider the case $Y=S^{1}-\{f(b)\}$ for any point $b$ in $X$.Assuming that $f^{-1}(Y)$ is not connected, we let $f^{-1}(Y)=A_{1} \cup A_{2}$ be a nontrivial separation. Then each $f\left(A_{i} \cup\{b\}\right)$ is connected. Since $f(b)$ is the only point common to $f\left(A_{1} \cup\{b\}\right)$ and $f\left(A_{2} \cup\{b\}\right)$, it must be an end point of each of them. Hence one can map each $A_{i} \cup\{b\}$ in a continuous one-one manner into $R$ with $b$ going to an end point of the image. "Piecing" these maps together, one obtains a continuous one-one map of $X$ into $R$, contrary to the hypothesis on $X$. Thus $X-\{b\}=f^{-1}(Y)$ is connected for all points $b$ in $X$.

In the general case, therefore, we may assume that $Y$ is a connected subset of $S^{1}-\{f(b)\}$ for some point $b$ in $X$ and apply (1.2).

Q.E.D.

(1.4) REMARK. If $X$ is a topological space with at least $n$ points and an $n-R-U$ system $\left\{f_{1}, \cdots, f_{n}\right\}$, where $n \geqq 2$, then $X$ is Hausdorff. For the map which takes each point $x$ in $X$ to the point $\left(f_{1}(x), \cdots, f_{n}(x)\right)$ in $R^{n}$ is continuous and one-one. (Cp. [10, p. 611].) 
The method found in $[14$, p. 9] (cp. [6, p. 1026]) yields:

(1.5) Lemma. Let $X$ be a topological space with at least $n$ points, $n \geqq 2$. If there is an $n-R-U$ system on $X$, then the complement in $X$ of each $n-2$ points can be mapped in a continuous one-one manner into a one-sphere.

(1.6) THEOREM. Let $X$ be a topological space with at least $n$ points, $n \geqq 2$. If there is an $n-R-U$ system on $X$, then there is a continuous one-one map of $X$ into a one-sphere. Conversely, if there is a continuous one-one map of $X$ into a one-sphere, then there is an $n-R-U$ system on $X$ (provided that $n$ is odd when all such maps are onto).

Proof. Let $\left\{f_{1}, \cdots, f_{n}\right\}$ be an $n-R-U$ system on $X$.

We consider first the case where $X$ is connected and show that $X$ is the sum of two arc-like sets (i.e., sets which map in a continuous one-one manner onto arcs) which meet only at their "end point(s)." From this information a continuous one-one map of $X$ into a one-sphere is easily constructed.

Now by (1.4) $X$ is Hausdorff and consequently is infinite. From (1.2), (1.3) and (1.5) one can show that the complement in $X$ of any $n+1$ points is not connected. Hence there is a minimal finite set $Z$ in $X$ which disconnects $X$. Let $X-Z=A_{1} \cup A_{2}$ be a nontrivial separation. Then for $i=1,2, B_{i}=A_{i} \cup Z$ is connected and no proper subset of $Z$ disconnects $B_{i}$. Since $A_{j}, j \neq i$, is infinite, there is a continuous one-one map $f_{i}$ of $B_{i}$ into $R$. It is claimed that each point of $f_{i}(Z)$ is an end point of $f_{i}\left(B_{i}\right)$. From this fact it is easy to see how $X$ is the sum of the two arc-like sets as described above.

To prove the claim, we first consider the case where $Z$ contains more than one point. Now (1.2) and the remarks made above about $Z$ and $B_{i}$ imply that no single point of $f_{i}(Z)$ can disconnect $f_{i}\left(B_{i}\right)$. Hence in this case each point of $f_{i}(Z)$ is an end point of $f_{i}\left(B_{i}\right)$. Next we consider the case where $Z$ is a single point. If $f_{i}(Z)$ in this case were not an end point of $f_{i}\left(B_{i}\right)$, then by (1.2) $A_{i}$ would not be connected. We shall deduce from this that $X$ contains a connected subset containing the point $Z$, which maps in a continuous one-one manner into $R$ but which is disconnected by $Z$ into at least three components. (In view of (1.2) this is an impossible situation and thus the claim is proved.) To obtain such subset, we choose, for $j \neq i$, a connected compact neighborhood $C_{j}$ of $f_{j}(Z)$ in $f_{j}\left(B_{j}\right)$ such that $f_{j}\left(B_{j}\right)-C_{j}$ is infinite and let $Z_{i}=A_{i} \cup f^{-1}\left(C_{j}\right)$. Then $Z_{i}$ would be the desired subset. From the remarks made above this completes the proof for the case where $X$ is connected.

We now consider the case where $X$ is not connected. A straightforward proof which makes use of the result just established and of (1.5) will show that either $X$ can be mapped into a one-sphere as desired or else $n \geqq 3$ and there is a nontrivial separation $X=A \cup B$, where $A$ contains at most $n-2$ points, where $B$ is a connected set which can be mapped in a continuous one-one manner into a 
one-sphere and where all such maps are onto. It remains then to show that the latter alternative is impossible. The method, mutatis mutandis, will be that common to [10], [14] and [15].

Assume then that $X=A \cup B$ as described. Let $f$ be a continuous one-one map of $B$ onto the unit circle in the complex plane. For $k=1, \cdots, n$, and for each $s$ in $[0,1]$ let

$$
x_{k}(s)=f^{-1}(\exp \{2 \pi i[k+s](1 / n)\}),
$$

i.e., for each such $s$, the points $x_{k}(s)$, in cyclic order, are the inverse images of vertices of a regular $n$-gon. By the hypothesis on $B$ and (1.3), if $Y$ is a connected subset of $[0,1] x_{k}(Y)$ is connected for all $k$. Thus the space

$$
Z=\underset{1 \leqq k \leqq n}{\mathbf{X}} x_{k}\{[0,1)\} \cup \underset{1 \leqq k \leqq n}{\mathbf{X}} x_{k}\{(0,1]\}
$$

is connected. If we define a map $g$ of $Z$ into $R$ by

$$
g\left\{\left(x_{1}\left(s_{1}\right), \cdots, x_{n}\left(s_{n}\right)\right)\right\}=\operatorname{det}\left\|f_{j}\left(x_{k}\left(s_{k}\right)\right)\right\|,
$$

then $g$ is continuous on $Z, g(Z)$ is connected, and by the definition of an $n-R-U$ system $g(Z) \subset R-\{0\}$. But we have

$$
g\left\{\left(x_{1}(0), \cdots, x_{n}(0)\right)\right\}=(-1)^{n-1} g\left\{\left(x_{1}(1), \cdots, x_{n}(1)\right)\right\}
$$

(for, roughly speaking, in changing the value, one permutes the vertices of the $n$-gon by a rotation through an angle of $2 \pi / n)$. We conclude that $n$ must be $o d d$. Now, by hypothesis there is a point $x_{0}$ in $A$. Then, in a way similar to that above, for each $s$ in $[0,1]$ we let the points $x_{k}(s), k=1, \cdots, n-1$, be the inverse images of the vertices of a regular $(n-1)$-gon while $x_{n}(s)=x_{0}$. Defining the space $Z$ and the map $g$ in formally the same manner as above, we conclude from a similar argumentation and from the fact that $n \geqq 3$ that $n$ must also be even. This contradiction gives the desired result.

To prove the converse, let us assume that there is a continuous one-one map $f$ of $X$ into a one-sphere $S^{1}$. If $f$ is onto, we may identify $S^{1}$ with the real numbers modulo $2 \pi$ and we have that $\{1, \cos f(x), \sin f(x), \cdots, \cos r f(x), \sin r f(x)\}$ is a $(2 r+1)-R-U$ system for each $r \geqq 1$. If all such maps $f$ are onto, then an argument similar to that given in the preceding paragraph will show that the order of any unisolvent system on $X$ must be odd. If there is a continuous one-one map of $X$ into $S^{1}$ which is not onto, assume that $f$ is such a map and identify $f(X)$ with a subspace of $R$. Then $\left\{1, f, \cdots, f^{n-1}\right\}$, where $f^{r}(x)=(f(x))^{r}, r=0,1, \cdots, n-1$, is an $n-R-U$ system on $X$.

Q.E.D.

2. Conditions for homeomorphism. What (1.6) tells us in general is that any subset of a one-sphere with any topology that contains the usual relative topology of that subset has an $n-R-U$ system for all suitable $n \geqq 2$ and that only such spaces have such systems. The natural question to ask is: When is the topology of these spaces exactly the relative topology? Several sufficient conditions for 
this are given below and examples follow to show the difficulty in finding conditions that are both necessary and sufficient.

(2.1) THEOREM. Let $X$ be a connected, locally connected space with at least $n$ points, $n \geqq 2$. If there is an $n-R-U$ system on $X$, then $X$ is topologically imbeddable into a one-sphere.

Proof. Assume that there is an $n-R-U$ system on $X$. Then by (1.6) there is a continuous one-one map $f$ of $X$ into a one-sphere $S^{1}$.

We consider first the case where $f$ is not onto and show that it is an open map and therefore a homeomorphism. For this it will suffice to prove that for any connected, open, proper subset $W$ of $X, f(W)$ is open in $f(X)$. Assume there is some subset $W$ of $X$ as described, such that $f(W)$ is not open in $f(X)$. Then $f(W)$ is either compact or is a half-open interval with its end point not an end point of $f(X)$. If $f(W)$ were compact, $W$ would be open and closed in $X$, contrary to the connectivity of $X$. Thus $f(W)$ must be a half-open interval as described. But then, considering $f(X)$ as a subspace of $R$, it is not difficult to find an open and closed proper subset $H$ of $X$ containing $W$, e.g., if $f(X)=[a, b]$ and $f(W)=[c, d)$, with $a<c<d \leqq b$, then $H=f^{-1}([c, b])=W \cup f^{-1}((c, b])$ would do.

We consider next the case where $f$ is onto. The argument proceeds as follows: either $f$ is a homeomorphism or there is a continuous one-one map of $X$ onto a proper subset of $S^{1}$ (which by the argument given above would be a homeomorphism). If then $f$ is not a homeomorphism, by an argument similar to that given above, there is a connected, open, proper subset $W$ of $X$ such that $f(W)$ is a half-open interval in $S^{1}$. Let $f(b)$ be the end point of $f(W)$. From the properties of $W$ and $f(W)$, it is not difficult to see that a continuous one-one map of $X$ onto a proper subset of $S^{\mathbf{1}}$ can be constructed by "opening up" $f(X)$ at the point $f(b)$. The image of $X$ will then be a half-open interval.

Q.E.D.

(2.2) COROLlaRY. Let $X$ be a connected, locally compact space with at least $n$ points, $n \geqq 2$. If there is an $n-R-U$ system on $X$, then $X$ is topologically imbeddable into a one-sphere.

Proof. Assume that there is an $n-R-U$ system on $X$. We shall show that $X$ is locally connected and the result will then follow from (2.1). Assume that there is a point $x_{0}$ at which $X$ is not locally connected. Let $V$ be a compact neighborhood $X$. Now by (1.6) there is a continuous one-one map $f$ of $X$ into a one-sphere. Then $V$ is homeomorphic via $f$ to a separable metric space and one may use the proofs found in [18, pp. 18-19], with the necessary changes, to obtain a sequence of pairwise disjoint, compact, nondegenerate, connected sets $M_{r}, r \geqq 0$, such that $x_{0} \in M_{0}$ and $\lim _{r \rightarrow \infty} M_{r}=M_{0}$. Letting $f(y)$ be an interior point of $f\left(M_{0}\right)$ and choosing $y_{r} \in M_{r}, r \geqq 1$, such that $\lim _{r \rightarrow \infty} y_{r}=y$, we have $\lim _{r \rightarrow \infty} f\left(y_{r}\right)=f(y)$. This is impossible since $f\left(M_{0}\right)$ is a neighborhood of $f(y)$ but contains none of the $f\left(y_{r}\right)$. Thus $X$ is locally connected.

Q.E.D. 
Local compactness alone, in the present context, is not enough to insure that a space be homeomorphic with a subspace of a one-sphere (cf. examples below). However, if a locally compact space is homeomorphic to such a subspace, it must be $\sigma$-compact. That condition in the present context is also sufficient, for

(2.3) THEOREM. Let $X$ be a locally compact, $\sigma$-compact space with at least $n$ points, $n \geqq 2$. If there is an $n-R-U$ system on $X$, then $X$ is topologically imbeddable into a one-sphere.

Proof. Assume there is an $n-R-U$ system $\mathscr{F}$ on $X$. We shall show first that $X$ is separable metric. By (1.4) $X$ is Hausdorff. Then since by hypothesis it is locally compact, it is also regular. Moreover, because of $\sigma$-compactness, we have by [16, pp. 16-17] that $X=\bigcup_{r \geqq 1} X_{r}$, where for each $r, X_{r}$ is open and $\bar{X}_{r}$ is compact. Via $f, X_{r}$ is therefore homeomorphic to a second countable space and hence is itself second countable. It follows that $X$ is second countable and separable metric.

In view of (2.2) we may assume that $X$ is not connected. It is claimed then that each nondegenerate component of $X$ is homeomorphic to an arc with or without end point(s). This follows easily from (2.2) and the proof in (1.6) for the case when $X$ is not connected, since each such component $D$ is infinite and locally compact and $\mathscr{F}$ restricted to $D$ is an $n-R-U$ system on $D$.

For a separable metric space with such components, the following conditions are necessary and sufficient that it be topologically imbeddable into $R$ (cf. [5]):

(a) if $x$ is a point-component of $X, \operatorname{dim}_{x} X=0$,

(b) if $x$ is an end point of an arc-component $D$ of $X, \operatorname{dim}_{x}[(X-D) \cup\{x\}]=0$, and

(c) if $x$ is an interior point of an arc-component $D$ of $X$, then $x$ is not a limit point of $(X-D)$.

(Note that if $Y$ is a subspace of $X$ and $x$ is a point of $Y$, then $\operatorname{dim}_{x} Y$ denotes the Urysohn-Menger dimension of $Y$ at $x[8, \mathrm{pp} .4,24]$.)

Now these properties hold in $X$. Property (c) can easily be proved by using a method similar to that contained in the proof of (2.2). Properties (a) and (b) are essentially local and thus it will be sufficient to show that they hold on the intersection of a compact neighborhood of the point $x$ with the sets in question. If one chooses a compact neighborhood of $x$ that maps via $f$ homeomorphically onto a proper subset of the one-sphere, the proof is complete for on such subsets of a one-sphere the properties do hold.

Q.E.D.

(2.4) REMARK. A natural setting for $\sigma$-compactness does arise. For assume that $X$ (is locally compact and) has at least $n$ points and an $n-R-U$ system $\mathscr{F}(n \geqq 2)$. For each $f$ in $\mathscr{C}(X)$ we have $X=\bigcup_{r \geqq 1}\{x:|f(x)| \geqq(1 / r)\} \cup\left\{f^{-1}(0)\right\}$. Then, since for each nonzero $f$ in the span of $\mathscr{F}$ over $R f^{-1}(0)$ contains at most $n-1$ points, $X$ is $\sigma$-compact when one such $f$ is in $\mathscr{C}_{0}(X)$. (Cp. [4].)

We are now in a position to answer Phelps' question: 
(2.5) THEOREM. Let $X$ be a locally compact Hausdorff space. If for some $n \geqq 2, X$ admits an $n$-dimensional Haar subspace in $\mathscr{C}_{0}(X)$, then $X$ has at least $n$ points and is topologically imbeddable into a one-sphere. Conversely, if $X$ has at least $n$ points $(n \geqq 2)$ and is topologically imbeddable into a one-sphere, then $X$ admits an $n$-dimensional Haar subspace in $\mathscr{C}_{0}(X)$ (provided that $n$ is odd if $X$ is a one-sphere).

Proof. Assume that for some $n \geqq 2, X$ admits in $\mathscr{C}_{0}(X)$ an $n$-dimensional Haar subspace $\mathscr{M}$. Let $\mathscr{F}$ be a basis over $R$ for $\mathscr{M}$. Since $\mathscr{F}$ is linearly independent, $X$ must have at least $n$ points. By Phelps' result (cf. introduction), $\mathscr{F}$ must be an $n-R-U$ system on $X$. Since $\mathscr{F} \subset \mathscr{C}_{0}(X)$, by (2.4) $X$ is $\sigma$-compact. Thus by (2.3) $X$ is topologically imbeddable into a one-sphere.

Conversely, assume that $X$ has at least $n$ points $(n \geqq 2)$ and is topologically imbeddable into a one-sphere. We shall construct an $n-R-U$ system on $X$ in $\mathscr{C}_{0}(X)$ (takinginto account the limitations noted in the statement of the theorem). If $X$ is compact, $\mathscr{C}_{0}(X)=\mathscr{C}(X)$ and the systems and proof found in (1.6) will give the desired result. If $X$ is not compact, we may topologically imbed $X$ into a compact interval $Y$ in $R$. For the rest of this proof $X$ will be identified with its image in $R$. Let $p$ be the usual metric for $R$ and define $d(x)=p(x, \bar{X}-X)$ for $x \in X$. Thus $d$ is in $\mathscr{C}(X)$. Since $X$ is locally compact, $X-X$ is compact (cf., e.g., $[9$, p. 50]) and thus $d(x)>0$ for all $x \in X$. For each $\varepsilon>0, Y-\{x: d(x) \geqq \varepsilon\}$ $=[Y-X] \cup\{y \in Y: p(y, X-X)<\varepsilon\}$ and is thus open in $Y$. Hence $\{x: d(x) \geqq \varepsilon\}$ is compact and $d$ is in $\mathscr{C}_{0}(X)$. Let $\mathscr{F}=\left\{f_{r}: r=0,1, \cdots, n-1\right\}$, where $f_{r}(x)=d(x) \cdot x^{r}$ for each $x$ in $X$. Then $\mathscr{F}$ is an $n-R-U$ system on $X$ in $\mathscr{C}_{0}(X)$. The rest follows from Phelps' result.

Q.E.D.

(2.6) Remarks. We give here some examples of spaces which have $n-R-U$ systems for all $n \geqq 2$ but which are not homeomorphic with any subspace of a one-sphere.

Firstly, consider the set $X$ of real numbers with the smallest topology that contains allthe open sets of the usual topology of $R$ and the set $R_{0}$ of rational numbers. The space is connected but neither locally compact nor locally connected. It is also separable but not metrizable.

Secondly, let $A$ be a locally compact subset of $R$ (with its usual topology) such that $B=R-A$ is uncountable. Let $X$ be the set of all real numbers with the smallest topology that contains the open sets of $A$ (with its usual relative topology) and the discrete topology on $B$. The space is locally compact, not connected, metrizable but not separable.

Lastly, let $X$ be the subspace of the Euclidean plane defined by $X=\{(x, 0): x \leqq 0\} \cup\{(x, \sin (1 / x)): x>0\}$. The space is connected, separable metric, projects in a continuous one-one manner onto $R$ but fails to be locally compact (though it is $\sigma$-compact). (Cp. $[17$, p. 196].) The removal of a certain countable collection of arcs from this example will yield a subspace which is, 
of course, still separable metric and maps in a continuous one-one manner into $R$, and whose components are now locally compact, but which still fails to be homeomorphic to any subspace of a one-sphere.

Thus this paper leaves unsolved the problem of finding necessary and sufficient conditions for a space with at least $n$ points and an $n-R-U$ system $(n \geqq 2)$ to be homeomorphic to a subspace of a one-sphere, i.e., conditions which take full advantage of the properties of an $n-R-U$ system.

\section{REFERENCES}

1. N. I. Achieser, Theory of approximation, Ungar, New York, 1956.

2. S. N. Bernstein, Leçons sur les propriétés extrémales et la meilleure approximation des fonctions analytiques d'une variable réelle, Gauthier-Villars, Paris, 1926.

3. R. C. Buck, Linear spaces and approximation theory, On Numerical Analysis, pp. 11-23, Univ. of Wisconsin Press, Madison, Wis., 1959.

4. - - Bounded continuous functions on a locally compact space, Michigan Math. J. 5 (1958), 95-104.

5. Leon W. Cohen, A characterization of those subsets of metric separable space which are homeomorphic with subsets of the linear continuum, Fund. Math. 14 (1929), 281-303.

6. Philip C. Curtis, Jr., N-parameter families and best approximation, Pacific J. Math. 9 (1959), 1013-1027.

7. Alfred Haar, Die Minkowskische Geometrie und die Annäherung an stetige Funktionen, Math. Ann. 78 (1918), 294-311.

8. W. Hurewicz and H. Wallman, Dimension theory, Princeton Univ. Press, Princeton, N. J., 1948.

9. C. Kuratowski, Topologie. II, Monogr. Mat. Tom. XXI, Warsaw, 1950.

10. John C. Mairhuber, On Haar's theorem concerning Chebychev approximation problems having unique solutions, Proc. Amer. Math. Soc. 7 (1956), 609-615.

11. Th. Motzkin, Approximation by curves of a unisolvent family, Bull. Amer. Math. Soc. 55 (1949), 789-793.

12. R. R. Phelps, Uniqueness of Hahn-Banach extensions and unique best approximation, Trans. Amer. Math. Soc. 95 (1960), 238-255.

13. I. J. Schoenberg, On the question of unicity in the theory of best approximation, Ann. New York Acad. Sci. 86 (1960), 682-692.

14. I. J. Schoenberg and C.-T. Yang, On the unicity of solutions of problems of best approximation, Ann. Mat. Pura Appl. Ser. IV 54 (1961), 1-12.

15. K. Sieklucki, Topological properties of sets admitting the Tschebycheff systems, Bull. Acad. Polon. Sci. Sér. Sci. Math. Phys. Astronom. 6 (1958), 603-606.

16. H. E. Vaughan, On locally bicompact spaces, Fund. Math. 31 (1938), 15-21.

17. A. J. Ward, The topological characterization of an open linear interval, Proc. London Math. Soc. (2) 41 (1936), 191-198.

18. Gordon T. Whyburn, Analytic topology, Amer. Math. Soc. Colloq. Publ.Vol. 28, Amer. Math. Soc., Providence, R.I., 1942.

University of Pennsylvania,

Philadel phia, Pennsylvania

WOODSTOCK COLLEGE, WOODSTOCK, MARYLAND 